\title{
Lactancia Materna: \\ Iniciación, beneficios, problemas y apoyo
}

\section{Breastfeeding:}

\section{Initiation, benefits, problems and support}

Pág. 105,118

Recibido: 06-07-2020

Aceptado: 10-09-2020

Dra. Mónica Solano Pochet ${ }^{1}$

1. Médico General. Investigador Independiente, Sustituciones Urgencias Hospital Clínica Bíblica. San José, Costa Rica.

\section{RESUMEN:}

La lactancia materna (LM) es el método preferido y es la fuente nutricional óptima. Se recomienda LM exclusiva los primeros 6 meses, posteriormente acompañada por alimentación complementaria por lo menos hasta los primeros 2 años. Existen factores parentales que influyen en el inicio y en el mantenimiento. La leche humana es rica nutricionalmente, tiene propiedades inmunológicas, antimicrobianas y de desarrollo. La iniciación y mantenimiento se ven influenciados por el ambiente hospitalario, social, cultural y familiar. Existen 10 pasos recomendados para que un hospital sea promotor. Se debe dar a demanda y se debe evaluar la ingesta adecuada. Existen múltiples beneficios a corto y largo plazo asociados para la salud del niño y la madre, la economía, sociedad y los sistemas de salud. Puede prevenir el riesgo de enfermedades agudas y crónicas.
La LM también tiene influencia sobre la madre y ocasiona un incremento en los requerimientos nutricionales. Existen problemas comunes como ingesta inadecuada, dolor, infecciones, secreción, sobreproducción e ictericia neonatal que pueden derivar de la LM, pero con manejo adecuado pueden resolverse y no ser impedimento. Se deben valorar las precauciones y contraindicaciones para LM en cada paciente, como infección materna, madres VIH positivas, uso de sustancias maternas y enfermedades infantiles. Se debe promocionar y apoyar la LM, educando sobre la manera adecuada para hacerlo, sus beneficios y evacuando dudas, aprovechando cada consulta.

\section{PALABRAS CLAVE:}

Lactancia materna, lactancia materna exclusiva, leche humana, iniciación, soporte, beneficios, problemas, destete. 
Abreviaciones: Lactancia Materna $=$ LM, INTRODUCCIÓN Lactancia Materna Exclusiva $=$ LME.

\section{ABSTRACT:}

Breastfeeding (BF) is the preferred method and is the optimal nutritional source. Exclusive $\mathrm{BF}$ is recommended for the first 6 months, subsequently accompanied by solid foods for at least the first 2 years. There are parental factors that influence the initiation and maintenance. Human breast milk is nutritionally rich, has immunological, antimicrobial and developmental properties. Initiation and maintenance are influenced by the hospital, social, cultural and family environment. There are 10 recommended steps for hospitals to promote BF. The infant should be given on demand and appropriate intake should be evaluated. There are multiple short and long-term benefits associated for the child's and mother's health, the economy, society and health systems. It can prevent the risk of acute and chronic diseases. Also influences the mother and cause increase in nutritional requirements. There are common problems such as inadequate intake, pain, infections, discharge, overproduction and neonatal jaundice that can come from BF, but with proper management they can be resolved and not be an issue. Precautions and contraindications must be assessed in each patient, such as infection, HIV positive mother, use of maternal substances and childhood illness. BF should be promoted and supported, educating on the appropriate way of doing it, its benefits and evacuating doubts, taking advantage of each consult.

\section{KEY WORDS:}

Breastfeeding, exclusive breastfeeding, human breast milk, initiation, support, benefits, problems, weaning.

La lactancia materna (LM) es el método preferido universalmente $(1,4)$ y es la fuente nutricional óptima en los primeros 6 meses de vida $(1,2,5,9,10,11,12)$. La AAP, AAFP, ACOG, OMS y CPS recomiendan LM exclusiva (LME) los primeros 6 meses $(1,5,9,10,11,12)$, seguida por LM con alimentación complementaria en el primer año de vida o más allá por los beneficios $(1,5,6)$. La OMS recomienda LM por lo menos hasta los primeros 2 años $(5,6,13)$. En niños tiene múltiples beneficios nutricionales, sobre la función gastrointestinal, contiene sustancias bioactivas que protegen contra enfermedades infecciosas y otorga bienestar psicológico $(2,8,11)$. Estudios epidemiológicos sugieren que la leche humana contribuye a la protección de enfermedades inflamatorias, autoinmunes y malignas en la infancia, con impacto a largo plazo en la función inmune (2). En las madres también se dan beneficios (8). Las madres y niños sin LM tienen mayores riesgos de salud (4), la LM subóptima se asocia a riesgo infantil aumentado de morbimortalidad con aumento de riesgo para condiciones crónicas (5). Cualquier cantidad recibida de LM es mejor que nada, pero los beneficios se aumentan a mayor duración (6). La capacidad materna para producir leche en cantidad y calidad suficiente para el crecimiento infantil es bastante resistente a la deprivación nutricional materna. Las mujeres que dan LM tienen aumentadas las demandas nutricionales (7). Para muchas mujeres las dificultades para la lactancia resultan en terminación previo al periodo recomendado. Sin embargo, con soporte, recomendaciones y tratamientos adecuados muchos de estos problemas se pueden solucionar y la LM se puede sostener por periodos más prolongados (4). Las madres de niños prematuros encuentran una variedad de barreras y retos para la LM que resultan en 
una disminución de LM comparado con niños a término, se debe hacer énfasis en lograr romper estas barreras para beneficio del niño (12). El soporte materno e infantil durante la estancia hospitalaria y posterior al egreso junto a la educación y soporte pre y posnatal incrementan la duración y el éxito $(6,10)$.

\section{METODOLOGÍA}

Para la creación de este artículo, se consultaron bases de datos de UpToDate, PubMed, Clinical Key, Elsevier, The Cochrane para buscar artículos recientes sobre el tema en revisión. De los artículos encontrados, se seleccionaron 13 referencias bibliográficas. Los criterios de inclusión utilizados para la búsqueda fueron: A) Periodo de tiempo comprendido entre 2012 a 2020. B) Idioma inglés y español. Para esto, se utilizaron términos de búsqueda como: "Breastfeeding", combinando con "Initiation" "Prevention", "Problems", "Weaning", "Support", "Benefits".

\section{EPIDEMIOLOGÍA}

A pesar de las recomendaciones mundiales para promover $\mathrm{LME}$, solo $35 \%$ de los recién nacidos a nivel mundial la reciben después de 3-4 meses posteriores al nacimiento (13). En EEUU $75 \%$ inicia LM, pero este porcentaje se afecta significativamente por diferencias culturales y sociodemográficas, variando desde $30 \%$ a $80.6 \%$ (1). Existen problemas éticos, prácticos y epidemiológicos para los estudios de LM $(1,2)$. Las madres que amamantan usualmente practican actividades que promueven la salud, son niños con distintas clases sociales, que van a guarderías por periodos distintos, las madres que piensan que sus hijos tienen alto riesgo para alergias y las madres de niños que comienzan a mostrar signos de enfermedad tienden a dar
LM por periodos más prolongados. Existen variaciones en la duración y el patrón, por lo general no se interrumpe repentinamente, es un proceso gradual que puede tardar desde días hasta meses haciendo difícil evaluar la dosis de exposición (2). Existen factores que influencian la continuidad de LM como falta de confianza, dolor, problemas de succión, grietas, infecciones, ingurgitación, mastitis, llanto del bebé, tipo de nacimiento, contacto piel con piel, mala experiencia previa, comentarios de familiares 0 amigos, trabajo, nivel socioeconómico y educativo $(1,13)$. La mayor parte de los abandonos a la LM se dan durante las primeras 4 semanas y entre los 3-4 meses de vida, los cuales coinciden con el retorno de la madre al trabajo (13).

Factores Parentales: Para poder promover la $L M$, es necesario entender que factores son importantes para los padres al decidir LM sobre fórmula, cuáles son los que la impiden y las razones para terminarla tempranamente (9). Factores asociados al fallo para iniciar LM: $<25$ años, madre soltera, bajo nivel de educación, fumado, bajo peso o prematuridad del neonato, pobreza, embarazo no deseado, depresión postparto, regreso temprano al trabajo. Factores asociados al fallo del mantenimiento de LM: Dificultades para la lactancia y percepción materna que la LM no cumple las necesidades de su hijo o de suministro inadecuado, dolor en pezones, falta de confianza propia, depresión, regreso al trabajo (9).

\section{COMPONENTES DE LA LECHE HUMANA}

La leche humana tiene propiedades inmunológicas y componentes biológicamente activos como macro-micronutrientes, células vivas, factores de crecimiento, compuestos antiinflamatorios, sustancias inmunológicas 
e inmunoprotectoras $(2,5)$. Muchos de estos factores son resistentes a las enzimas digestivas y son activos biológicamente en la superficie mucosa. Incluyen: (5) Actividad antimicrobiana: Inmunoglobulinas, IgA, lisozimas, enzimas antimicrobianas, lactoferrina, ácidos grasos libres, monoglicéridos, lipasa, mucinas, leucocitos, células madre y oligosacáridos con acción antimicrobiana y prebiótica que ayudan a proteger contra infecciones y el desarrollo de enterocolitis necrotizante (2,5). Actividad inmunomoduladora: interleukina-10, ácidos grasos poliinsaturados, acetilhidrolasa del factor activador de plaquetas, glicoconjugados, agonistas y antagonistas de respuesta inmune innata. Protegen de enterocolitis necrotizante $(2,5)$. Factores que promueven el desarrollo y funcionamiento gastrointestinal: proteasas, hormonas, factores de crecimiento, mediadores gastrointestinales, aminoácidos que estimulan el crecimiento del enterocito, influencia en desarrollo de flora intestinal (5).

\section{INICIACIÓN}

-Ambientehospitalario:Durantelahospitalización hay mayores posibilidades de iniciar LM si hay políticas que la promuevan $(10,11)$. La OMS y UNICEF desarrollar 10 pasos para que un hospital promueva LM: 1. Políticas hospitalarias que cumplan con el código internacional de marketing de sustitutos de leche humana, tener política de alimentación infantil escrita y que sea comunicada al personal y padres. 2. Entrenar a todo el personal con conocimientos, competencia y habilidades necesarias para apoyarla. 3. Informar a todas las embarazadas y su familia sobre la importancia, beneficios y manejo de la LM. 4. Facilitar el contacto piel con piel e iniciar LM tan pronto como sea posible. 5. Enseñar a las madres como dar lactancia y como mantenerla. 6 . No dar otros alimentos o bebidas a los recién nacidos a menos que sean médicamente indicados. 7. Permitir que las madres y niños permanezcan juntos 24 horas al día. 8. Alentar LM a demanda y ayudar a las madres a reconocer y responder las señales de hambre. 9. Aconsejar a las madres sobre los riesgos de chupones, pezoneras o chupetas. 10. Coordinar el egreso para que los niños y padres tengan acceso al apoyo y velar por el establecimiento de grupos de apoyo para LM y referir a las madres $(1,10)$. En la sala de parto se debe incentivar el contacto piel con piel, poner en contacto los labios del niño con los pezones de la madre y la LM se debe iniciar cuanto antes posible, idealmente en la primera hora.

La iniciación en cesáreas es menor a los partos vaginales. Los cuidados postparto incluyen educación y apoyo parental, evaluando LM e identificando posibles problemas. Posterior al egreso, se debe reevaluar a la madre y al niño 24-48 horas después $(10,11)$.

\section{- Mecánica de la alimentación:}

La lactogénesis ocurre en 2 etapas; I iniciación secretora en la segunda mitad del embarazo y II Activación secretora que ocurre después del nacimiento.

\section{Posición:}

La madre debe estar en una posición cómoda mientras amamanta, el niño se acomoda con la boca opuesta al pezón materno, con el cuello ligeramente extendido. La cabeza, hombros y cadera alineados o se puede colocar en posición de "fútbol" (10). Sello: Se debe formar un sello con los labios del niño alrededor del pezón y de una porción suficiente de tejido mamario que permite la extracción eficiente de leche. Se toma 
el pecho con una mano con 4 dedos debajo y el pulgar encima, el niño abre su boca y agarra el pezón completo y cuanto pueda de areola (10), deben haber $3-4 \mathrm{~cm}$ del tejido mamario en la boca del niño (6). La lengua del niño alarga el pezón y comprime los conductos lactíferos contra el paladar duro facilitando la extracción. Apariencia de un sello adecuado: Ángulo de $120^{\circ}$ entre el labio superior e inferior, eversión de los labios, la barbilla y nariz están en proximidad del pecho, mejillas se ven llenas, lengua visible debajo del pezón (10), se deben escuchar sonidos del niño tragando, patrón rítmico de succión y tragado (6). Se puede ver afectado por anquiloglosia o disfunción neurológica en el niño y pezones maternos invertidos $(6,10)$.

\section{- LM a demanda:}

A demanda significa que se da LM en respuesta a las señales de hambre del niño. Se ofrecen ambos pechos en cada toma, el primer pecho que se ofrece debe alternarse para que cada pecho reciba igual estimulación y drenaje. La frecuencia para amamantar es variable y depende de factores como suministro de leche humana, eficacia de las tomas previas y capacidad del lactante para modular su comportamiento. Las señales de hambre son cambios en el comportamiento, llevar las manos a la boca, succión de los puños y dedos, agitación, movimiento de extremidades y por último llanto. Se debe alimentar al inicio de los signos para que el niño esté menos agitado y poder hacer un sello adecuado. Durante la primera semana postparto o hasta que LM esté establecida, se debe amamantar al niño al mostrar signos de hambre o se debe despertar al niño para amamantarlo si han pasado 4 horas desde el inicio de la última toma. Signos de saciedad: el niño suelta el pecho, relaja los músculos faciales y las manos o se queda dormido (10).

\section{- Evaluación de la ingesta:}

Durante la primera semana el niño realiza 8-12 tomas diarias, a las 4 semanas 7-9 tomas diarias. La duración de las tomas depende de la eficiencia puede ser de 5-20 minutos por pecho, inicialmente es de 10-15 minutos por pecho y para el mes 8-10 minutos. En las primeras 24 horas orinan 1 vez, 2-3 veces en el segundo día, 4-6 en el tercer y cuarto día, al quinto día los niños orinan 6-8 veces por día.

Presentan heces transicionales los primeros 3 días, después del cuarto día defecan 3 o más veces por día y para el quinto día las heces son pastosas y color amarillo pálido. Durante las primeras 24 horas las tomas son de $6 \mathrm{~mL}$ por toma, día 3 son $30 \mathrm{~mL}$ y día 5 son $60 \mathrm{~mL}$. Los niños a término normalmente pierden $7 \%$ de su peso corporal al nacimiento en los primeros 3 a 5 días y lo recuperan 1-2 semanas después del nacimiento. Si el niño pierde $>7 \%$ de su peso o falla para recuperarlo se debe considerar la opción de ingesta inadecuada. Deben ganar $15-40 \mathrm{~g} /$ día o $105-210 \mathrm{~g}$ por semana. Se debe evaluar si los pechos maternos se llenan de leche y si hay alguna lesión.

La producción es dependiente de la estimulación y evacuación de los pechos para que el organismo a través de la prolactina produzca la cantidad de leche que el infante requiere, sin la evacuación adecuada el cerebro interpreta que no requiere más producción $(4,6,10)$.

La suplementación se debe reservar para pacientes que han perdido $>7 \%$ del peso de nacimiento a los 5-10 días, signos de deshidratación, <3 deposiciones fecales pequeñas por día y el suministro materno es limitado (10).

\section{-Lactancia Materna en Pretérminos:}


componentes estimuladores del crecimiento y

Prematuros deberían recibir leche por sus múltiples beneficios $(1,12)$. Muchos no pueden alimentarse del pecho inicialmente, pero la leche debe ser extraída, colectada y dada al niño por otros medios. LM depende del desarrollo de habilidades orales, de localizar el pecho materno y hacer un sello adecuado. Durante la hospitalización, la progresión de LM es monitorizada evaluando la ingesta por el peso en el lactante. Cuando el niño demuestra habilidad para consumir todas las tomas diarias se puede hacer un esquema a libre demanda modificado basado en una ingesta mínima de leche en 24 horas. Requieren suplementación de hierro (12).

\section{-Uso de chupeta:}

La evidencia actual sugiere que las chupetas no interfieren con el establecimiento o mantenimiento de la lactancia (10) y puede tener efecto protector contra el síndrome de muerte súbita infantil $(1,10)$.

\section{- Suplementos:}

Se puede suplementar con hierro oral profiláctico a partir del cuarto mes de edad en niños nacidos a término y con peso adecuado, para reforzar las reservas (1).

\section{BENEFICIOS}

\section{- Beneficios a corto plazo:}

Neuroconductuales: el contacto piel con piel ayuda a la adaptación y parece tener efecto analgésico. Función Gastrointestinal: estimula el crecimiento adecuado, desarrollo y función del sistema gastrointestinal e influencia el desarrollo de la flora intestinal, tiene motilidad gastrointestinal y factores protectores (5). Reduce el riesgo de gastroenteritis y diarrea un $64 \%(1,5,13)$, aumenta el tiempo de vaciado gástrico, aumenta la actividad de la lactasa intestinal y disminuye la permeabilidad intestinal en prematuros (5), reduce el riesgo de desarrollo de enterocolitis necrotizante un $58 \%$.

\section{Enfermedad respiratoria:}

reduce $72 \%$ el riesgo de enfermedad respiratoria y disminuye la severidad de bronquiolitis $74 \%$. Otitis media: la incidencia de otitis media y otitis media recurrente se reduce $23 \%$, pero más allá de 3 meses de LM se reduce $63 \%(1,5)$. Infección del tracto urinario: reduce el riesgo de infección urinaria y se cree que es por mayor contenido en la orina de oligosacáridos, lactoferrina e IgA secretora. Sepsis: disminuye el riesgo de sepsis neonatal (5). Síndrome de muerte súbita infantil: reduce $36 \%$ el riesgo y a mayor tiempo de LME mayor protección $(1,5)$. Mortalidad y hospitalización: reduce el riesgo (5).

\section{- Beneficios a largo plazo:}

Enfermedades agudas: efecto en reducir enfermedades agudas incluso cuando la LM ha sido descontinuada y parece aumentar a mayor tiempo lactando (5). Enfermedades Crónicas: LM reduce la incidencia de enfermedades como diabetes Mellitus tipo 1 y 2 un $30 \%$, síndrome inflamatorio intestinal se reduce $31 \%$, sibilancias, maloclusión y caries dentales, reduce $20 \%$ el riesgo de leucemia y linfoma, a mayor duración de LM reduce el riesgo de obesidad $15-30 \%$, hay cierta asociación a disminución en factores de riesgo cardiovascular, reduce el riesgo de enfermedad celiaca $52 \%$, se asocia a mejoras en el neurodesarrollo con mayor coeficiente intelectual a futuro, desarrollo cognitivo, función 
visual y función auditiva, y disminución del déficit de atención e hiperactividad y trastornos del espectro autista $(1,5)$. Impacto en el desarrollo de enfermedad alérgica: LME por 3-4 meses se asocia a disminución en el riesgo de asma, dermatitis atópica y eczema $27 \%$ en población de bajo riesgo y $42 \%$ en lactantes con historia familiar positiva $(1,2,13)$. El efecto en el desarrollo de rinitis alérgica es controversial, se ha encontrado asociación con reducción. LME en los primeros 4 meses puede disminuir el riesgo de alergia a la leche de vaca (2).

\section{-Beneficios económicos:}

Tiene beneficios económicos familiares y sociales. Beneficios sociales: Se realizó un análisis global siguiendo las recomendaciones de las OMS el cual estima la cantidad de muertes que se pueden prevenir anualmente: 595,379 muertes por diarrea y neumonía en niños de 6-59 meses de edad, 974,566 casos de obesidad, 98,243 muertes por diabetes mellitus 2 , cáncer de mama y ovárico.

Estos y otros efectos adversos de salud por falta de LM óptima se traducen en pérdidas globales económicas de $\$ 341.3$ billones anuales (8). Si el $90 \%$ de las madres en EEUU dieran LME por 6 meses se ahorrarían 13 billones anuales (1). Además, tiene beneficios ambientales al evitar el uso de agua para preparar la fórmula y su consumo, desperdicio de plástico, papel y metal de las botellas y empaques de la fórmula, emisiones de carbón asociadas con la producción, empaque, transporte y preparación de la fórmula.

\section{Beneficios familiares:}

Se ahorra el costo económico de la fórmula y costos médicos por enfermedades prevenibles tanto en las madres como en los niños (8).

\section{LACTANCIA EN LA MADRE}

\section{- Evaluación prenatal de los pechos:}

Se deben evaluar factores que pueden intervenir con la lactancia, como tejido mamario hipoplásico, anormalidades del pezón y cirugía mamaria (9).

\section{- Beneficios Maternos:}

Tiene beneficios maternos a corto y largo plazo $(1,8)$, como reducción de riesgo de pérdida sanguínea postparto al activar oxitocina $(1,8)$, involución uterina más rápida (1), amenorrea (1) y retraso en la ovulación posterior al nacimiento (8), el tiempo de retraso varía y depende de la frecuencia de LM (8), disminución del riesgo de cáncer de mama, ovárico y endometrial, prevención de enfermedad cardiovascular, disminución en el riesgo de Diabetes Mellitus tipo 2, la protección es mayor en aquellas con LM prolongada $(1,8)$, disminución en el riesgo relativo para artritis reumatoide (1).

El cambio de peso es muy variable (7), los estudios relacionados al retorno del peso previo al embarazo han sido inconclusos $(1,8)$. Se da pérdida mineral ósea durante la lactancia por reabsorción ósea, pero se da remineralización durante el destete y posterior a LM, no aumenta el riesgo de fracturas a largo plazo $(7,8)$.

\section{- Determinantes del volumen lácteo:}

contacto piel con piel al nacimiento, demanda del lactante, nutrición materna, vaciamiento de las mamas, fumado, estrés, ansiedad, fatiga y enfermedad materna, uso de anticonceptivos orales combinados (7).

- Efectos de la nutrición materna en la calidad 


\section{de la leche:}

LM requiere energía y nutrientes adicionales en la leche. La calidad de la leche humana por lo general es suficiente para el crecimiento infantil aún cuando la dieta materna es limitada $(7,9)$. El contenido de proteínas no suele afectar la leche. El contenido graso tiene mínimos efectos en la cantidad de los ácidos grasos en la leche. La concentración de vitaminas lipo e hidrosolubles es reducida en la leche si hay deficiencia en la madre y se aumentan con la suplementación. Contenido mineral es independiente de los niveles maternos (7).

\section{- Requerimientos nutricionales:}

El requerimiento calórico se basa en la edad, peso, altura y actividad, de los 0-6 meses postparto requieren $330 \mathrm{kcal} / \mathrm{día}$ más, de los 7-12 meses requieren $400 \mathrm{kcal} / \mathrm{día}$. Se recomiendan 71 g/día de proteína de los 0-6 meses y en promedio $1 \mathrm{~g}$ de proteína por $100 \mathrm{~mL}$ de leche producido. Los requerimientos de vitaminas lipo e hidrosolubles están aumentados. Los requerimientos de calcio, fósforo y magnesio son los mismos que en las mujeres no lactantes. Se necesitan $9 \mathrm{mg}$ de hierro por día, inferior a las no lactantes ya que la amenorrea reduce la pérdida de hierro. El requerimiento de Zinc y Selenio está aumentado (7). Muchos médicos recomiendan continuar con las vitaminas prenatales durante la lactancia (1).

\section{- Dietas especiales:}

En mujeres con sobrepeso $u$ obesas se debe restringir la ingesta de $500 \mathrm{kcal} /$ día y realizar ejercicio (7). Las dietas vegetarianas tienen más riesgo de deficiencia mineral, vitamínica y proteica, se puede suplementar con calcio, vitamina $D$ y $B 12$. Las que tienen dieta vegana deben tomar suplementos vitamínicos que contengan vitamina B12 $(7,9)$. No se recomienda evitar ciertas comidas para prevenir alergias en los lactantes. Se recomienda evitar el consumo de pescados con altas concentraciones de mercurio (7), pero no se niega el consumo de todos los pescados ya que el omega 3 es beneficioso para el lactante (1).

\section{- Embarazos subsecuentes:}

El embarazo no es una contraindicación para la lactancia, pero la ingesta calórica debe aumentarse. Duranteelembarazo la composición de le leche cambia y el suministro disminuye. La succión puede estimular contracciones (9).

\section{PROBLEMAS COMUNES}

- Ingesta inadecuada de leche humana: la ingesta inadecuada o la percepción de la misma, es la causa más común para terminación de LM. Se puede deber a falla para la extracción por el niño o insuficiente producción. El diagnóstico se hace clínicamente por la historia, disminución en excretas urinarias y fecales y pérdida de peso. Signos de ingesta inadecuada: pérdida de peso $>7 \%$, no hay sonidos audibles del niño tragando durante la alimentación, $<6$ pañales húmedos por día, $<3$ defecaciones por día, hipernatremia (sodio sérico $>150 \mathrm{mEq} / \mathrm{L}$ ), deshidratación, pueden llegar a presentar ictericia neonatal, cambios mínimos en los pechos maternos, dolor en los pezones maternos después de 30-60 segundos del inicio de la lactancia. El manejo se basa en optimizar la técnica, se debe evaluar si hay producción inadecuada o si hay pobre extracción. Evitar suplementar con fórmula o LM de donante ya que disminuye la producción, pero esta puede ser necesaria en ciertos casos para una nutrición adecuada. No se recomienda el uso de galactogogos $(4,6,10)$. 


\section{- Ictericia Neonatal:}

La LM se asocia con hiperbilirubinemia por 2 entidades distintas: falta de ingesta de leche humana o ictericia de la leche materna. La primera, se manifiesta con los signos de ingesta inadecuada de leche humana mencionados anteriormente y es la causa más frecuente de ictericia en la primera semana de vida. La segunda, la madre tiene una adecuada producción de leche, pero se da elevación de la bilirrubina indirecta por inhibición competitiva enzimática contra la bilirrubina indirecta en el hepatocito y circulación enterohepática $(4,10)$.

\section{- Dolor mamario y de pezones:}

El dolor puede ser ocasionado por succión o sello inadecuado, vasoconstricción, congestión mamaria, ductos obstruidos, galactoceles, infecciones, excesiva producción de leche, dermatitis, psoriasis. Se evalúa con la historia, examinación del niño y de los pechos maternos, y observación de la alimentación. El manejo incluye la prevención de lesiones y tratar los pezones lastimados, remover un poco de leche para aliviar la congestión, utilizar compresas tibias para facilitar la extracción, compresas frías al finalizar la toma, utilizar antiinflamatorios, tratar los trastornos mamarios y los problemas mecánicos en el lactante $(4,6)$.

\section{- Infecciones mamarias:}

Mastitis es una inflamación localizada de la mama que se asocia a fiebre, mialgias, dolor mamario y enrojecimiento, causada por congestión, pobre drenaje, ineficiente extracción o alimentaciones infrecuentes, sobreproducción láctea, trauma en pezón y presión en el pecho. Absceso mamario: es una colección localizada de pus usualmente precedida por mastitis, se manifiesta como la mastitis con dolor mamario, síntomas sistémicos con una masa palpable, fluctuante y suave. Infección candidiásica se manifiesta como dolor mamario desproporcionado a los hallazgos físicos, historia de candidiasis vaginal materna o candidiasis oral o del pañal en el lactante, piel brillante o escamosa en el pezón, cultivo positivo. Se trata con antifúngicos tópicos y en casos refractarios con antifúngicos orales (4).

\section{- Secreción sanguinolenta por el pezón:}

Algunas mujeres presentan descarga sanguinolenta durante los primeros días, es más común en el primer embarazo, se da por mayor vascularización de los ductos con el inicio de la producción láctea. Si continúa más allá de la primera semana se deben considerar otras causas, como pezones agrietados, mastitis y papiloma intraductal (4).

\section{- Sobreproducción láctea:}

Generalmente, la producción es determinada por la demanda infantil, pero en este caso la producción supera la demanda. En algunos casos la eyección láctea es muy fuerte y el lactante puede tener problemas para alimentarse, puede ahogarse, toser o tornarse irritable, pueden tener aumento o pérdida de peso. Se maneja con una posición más erecta o de lado para amamantar, reducción manual del flujo, estrategias alimentarias, compresas frías, medicamentos como galactogogos (4).

\section{- Ictericia Neonatal:}

la LM se asocia con hiperbilirrubinemia en 2 tipos de entidades; ictericia de la leche materna o ictericia por leche materna (4).

\section{Destete}


El destete es una decisión personal materna en su contexto social, influenciada por factores como embarazos, salud, trabajo y carrera profesional. El destete repentino no es recomendado. La madre puede experimentar congestión mamaria, rápido descenso de prolactina que puede aumentar síntomas depresivos y una especie de resfrío por reabsorción de productos de la leche. Se recomienda el destete rutinario, el cual es más fácil después de los 6 meses con el inicio de comidas sólidas ya que el niño mismo lo lidera al ir disminuyendo las tomas y el proceso gradual de destete comienza. Las estrategias incluyen dejar de dar alguna de las tomas cada 2-5 días, reduciendo la duración de cada toma y aumentando el tiempo entre cada toma. Es importante que durante el proceso la mamá mantenga la cercanía. Si sucede congestión mamaria se debe evitar extraer la leche ya que estimula la producción (4).

\section{CONTRAINDICACIONES Y PRECAUCIONES}

\section{-Infección materna:}

Mujeres infectadas por virus linfotrófico humano de células $T$ tipo I o II o brucelosis no tratada no deben dar LM. Las mujeres con lesiones herpéticas en los pechos no deben amamantar del lado afectado hasta que la lesión se resuelva y se deben cubrir las lesiones para que el bebé no entre en contacto. Las mujeres con tuberculosis activa no deben amamantar, pero si pueden dar leche extraída ya que tuberculosis no se transmite en la leche. Las madres que desarrollan varicela 5 días previos o 2 días posteriores al nacimiento deben ser separadas de sus hijos, pero se pueden alimentar con leche extraída ya que no hay transmisión a través de esta. Las madres con influenza H1N1 deben ser separadas de sus hijos hasta estar afebriles, pero la leche extraída si puede ser administrada. La transmisión por CMV se puede dar en niños de muy bajo peso al nacer, pero si la leche es pasteurizada se inactiva el virus, pero también daña los factores bioactivos y los nutrientes $(1,9)$. La hepatitis materna por virus de hepatitis B es compatible posterior a la inmunoprofilaxis. Se debe evitar administrar la vacuna para fiebre amarilla excepto en áreas con brotes (9).

\section{- Madres VIH positivas:}

La transmisión madre a hijo se puede dar intraútero, en el parto o postnatal a través de la LM. Se ha detectado virus en la leche humana y mayores índices de transmisión en áreas donde la alimentación es principalmente LM. Se sugiere que la transmisión es mayor en los primeros meses de vida. El principal factor para transmisión, es la viremia en la madre. Se asocian a incremento en el riesgo de transmisión niveles altos de ARN de VIH en plasma, niveles de ADN y ARN de VIH en la leche humana, madres inmunosupresas con conteos bajos de CD4 e infecciones en los pechos (3). La transmisión puede ser reducida con la administración de antiretrovirales maternos y profilaxis antiretroviral infantil (3). Se debe balancear el riesgo de transmisión de VIH y el de malnutrición e infecciones severas (3). Madres infectadas por VIH con posibilidades para sustituir LM por fórmula deben sustituirla. Si la sustitución no es posible, se recomienda LME con antiretrovirales por 6 meses y posteriormente junto con alimentación complementaria reduciendo el riesgo de contagio $(1,3,9)$.

\section{- Uso de sustancias maternas:}

Se contraindica LM en madres que utilizan drogas ilícitas como cocaína, fenciclidina y marihuana. Las madres dependientes de narcóticos 
pueden amamantar si se encuentran en un programa supervisado para el mantenimiento de metadona, VIH negativos y si tienen tamizaje negativo por drogas ilícitas $(1,9)$.

El alcohol puede inhibir la respuesta de la prolactina a la succión y afectar negativamente el desarrollo motor infantil, se puede consumir ocasionalmente pero no más de $0.5 \mathrm{~g} / \mathrm{kg}$ de la madre y se debe amamantar hasta 2 o más horas después de la ingesta para minimizar su concentración en la leche $(1,7)$.

Mujeres que lactan pueden tomar 2-3 tazas de bebidas cafeínadas por día (7). Fumar no es una contraindicación absoluta, pero debe ser desalentado ya que se asocia a síndrome de muerte infantil súbita y a alergia respiratoria $(1,9)$. La mayoría de fármacos son compatibles con la lactancia. Los medicamentos que pueden ser prescritos directamente al niño por lo general son seguros porque las dosis transmitidas por la leche son inferiores a las dosis terapéuticas.

Por lo general medicamentos con pobre biodisponibilidad oral no afectan al niño, los medicamentos con barrera para proteínas, con baja solubilidad lipídica o con alto peso molecular no pasan en la leche. El riesgo de toxicidad aumenta en niños prematuros y enfermos y es rara en $>6$ meses. La exposición se puede minimizar al administrar el medicamento posterior a amamantar y antes del sueño prolongado (4). No se recomienda la lactancia en madres recibiendo anfetaminas, agentes quimioterapéuticos, estatinas y ergotamina (1).

\section{- Factores infantiles:}

La galactosemia infantil y el déficit congénito de lactasa son una contraindicación absoluta para LM, otros errores innatos del metabolismo como fenilcetonuria pueden recibir LM parcial, pero los niveles de fenilalanina deben ser monitoreados y la LM ajustada $(1,9)$.

\section{PROMOCIÓN:}

\section{- Rol Médico:}

Todos los médicos deberían guiar y apoyar a los padres que eligen LM, promover LM como norma, conocer los principios y el manejo, desarrollar habilidades para evaluar si la LM es adecuada, promover políticas hospitalarias compatibles con la AAP, OMS y UNICEF para una LM exitosa, tienen un rol esencial en la educación prenatal, impulsando la LM durante la hospitalización, dando consejo y apoyo a la madre durante la hospitalización y posterior al egreso, se deben aprovechar las consultas postnatales para alentar la LM y se debe evitar entregar fórmulas $(1,9)$.

\section{- Apoyo y educación parental:}

La LM es un acto natural y aprendido que es posible para las madres al tener información adecuada, apoyo familiar, comunitario y de los sistemas de salud. La promoción, debe incorporar factores culturales y considerar los ambientes familiares (13). Parte del éxito depende de profesionales brindando educación y apoyo. Se recomiendan programas educativos pre y posnatales, que incluyan habilidades prácticas, material escrito y apoyo postparto con seguimiento. La presencia de padres comprometidos y educados con LM se asocia a mayor efectividad. El apoyo se debe extender por todo el tiempo que se brinde LM. Se debe evitar entregar fórmula o material promocional de fórmulas (9). En los trabajos, se debe brindar un ambiente amigable para las madres con espacios físicos y tiempo para extracción de 
leche (1). En muchos casos, las madres podrían no tener la información suficiente $\mathrm{o}$ haber decidido dar LM, por lo que se le deben explicar los beneficios. La mayoría de prematuros al inicio no pueden alimentarse directamente del pecho y sus madres necesitan instrucciones y educación para la extracción y almacenaje de la leche. Cuando el bebé ya puede alimentarse del pecho se debe enseñar a las madres la mejor manera para iniciar y enseñar el mejor manejo para LM posterior al egreso (12).

\section{CONCLUSIONES:}

La LM es un asunto de salud pública, debe ser promovida y apoyada. Se debe velar por la educación del personal de salud, materna y familiar para optimizarla. La LM es el método preferido para alimentación exclusiva en menores de 6 meses y en combinación con alimentación complementaria hasta los 2 años o más por sus múltiples beneficios a corto y largo plazo en el lactante, en la madre, economía y en los sistemas de salud. Se debe fomentar un ambiente hospitalario que propicie el inicio y el mantenimiento de la LM. La capacidad materna para producir leche es bastante resistente a la deprivación nutricional, pero las mujeres que dan LM tienen aumentadas las demandas nutricionales. Existen ciertos problemas relacionados a la lactancia, pero en su mayoría con la guía adecuada se pueden superar. Hay una serie de contraindicaciones y precauciones las cuales deben ser valoradas en cada paciente.

El destete se debe hacer rutinaria y respetuosamente. En cada consulta después del nacimiento, se debe evaluar el estado de la LM y se debe aprovechar para evaluar, abarcar dudas y apoyar.

\section{BIBLIOGRAFÍA}

1. Arthur L Eidelman, MD, Richard J Schanler, MD. Breastfeeding and the use of human milk. Pediatrics. 2012 March; 129 (3): 827-841. DOI: 10.1542/peds.2011-3552 Recuperado de: http:// pediatrics.aappublications.org/content/129/3/e827.full.html

2. David M Fleischer, MD. The impact of breastfeeding on the development of allergic disease. UpToDate. 2019 Apr 5: 1-16. Recuperado de: https://www.uptodate.com/contents/the-impact-ofbreastfeeding-on-th...earch_result\&selectedTitle=8 150\&usage_type=default\&display_rank=8

Grace John-Stewart, MD. Prevention of HIV transmission during breastfeeding in resource-

3. limited setting. UpToDate. 2019 Oct 18: 1-37. Recuperado de: https://www.uptodate.com/ contents/prevention-of-hiv-transmission-du...arch_result\&selectedTitle=12 150\&usage_ type $=$ default\&display_rank $=12$

4. Jeanne Spencer, MD. Common problems of breastfeeding and weaning. UpToDate. 2020 Jan 28: 1-50. Recuperado de: https://www.uptodate.com/contents/common-problems-ofbreastfeeding...arch_result\&selectedTitle=1 150\&usage_type=default\&display_rank=1

5. Joan Y Meek, MD, MS. Infant benefits of breastfeeding. UpToDate. 2020 Jun 4: 1-26. Recuperado de: https://www.uptodate.com/contents/infant-benefits-of-breastfeeding/ print?search=breast\%20feeding\&topicRef=4996\&source=see_link 
6. Katie L Westerfield, DO, Kristen Koenig, MD, Robert Oh, MD, MPH. Breastfeeding: Common questions and answers. Am Fam Physician. 2018 Sep 15; 98 (6): 368-373C. Recuperado de: https://www.aafp.org/afp/2018/0915/ p368

7. Nancy F Butte, PhD, Alison Stuebe, MD, MSc. Maternal Nutrition during lactation. UpToDate. 2020 Apr 20: 1-35. Recuperado de: https://www.uptodate.com/contents/maternal-nutritionduring-lactation/print?search=breast\%20feeding\&topicRef=4986\&source=see_link

8. Rafael Pérez-Escamilla, PhD, Sofía Segura-Pérez, MS, RD. Maternal and economic benefits of breastfeeding. UpToDate. 2019 Nov 26: 1-12. Recuperado de: https://www. uptodate.com/contents/maternal-and-economic-benefits-o...eding/print?search=breast $\% 20$ feeding\&topicRef=5013\&source $=$ see_link

9. Richard J Schanler, MD, Debra C Potak, RN, BSN, IBCLC. Breastfeeding: Parental education and support. UpToDate. 2019 Nov 1: 1-24. Recuperado de: https://www. uptodate.com/contents/breastfeeding-parental-education...upport/print?search=breast $\% 20$ feeding\&topicRef $=4960$ \&source $=$ see_link

10. Richard J Schanler, MD, Debra C Potak, RN, BSN, IBCLC. Initiation of breastfeeding. UpToDate. 2020 Jan 1: 1-33. Recuperado de: https://www.uptodate.com/contents/initiation-of-breastfeeding/ print?search=breast\%20feeding\&topicRef=4996\&source=see_link

11. Sarah S Cohen, PhD, Dominik D Alexander, PhD, Nancy F Krebs, MD, Bridget E Young, PhD, CLC, Michael D Cabana, MD, MPH, Peter Erdmann, PhD et al. Factors associated with breastfeeding initiation and continuation: A Meta-Analysis. The Journal of Pediatrics. 2018 Dec; 203: 190-196. Recuperado de: https://doi.org10.1016/j.jpeds.2018.08.008

12. Steven A Abrams, MD, Nancy M Hurst, PhD, RN, IBCLC. Breastfeeding the preterm infant. UpToDate. 2020 Jan 28: 1-23. Recuperado de: https://www.uptodate.com/contents/breastfeedingthe-preterm-infant/print?search=breast\%20feeding\&topicRef=4978\&source=see_link

13. Susana Martín-Iglesias, M Jesús Santamaría-Martín, Ahinoa Alonso-Álvarez, Milagros RicoBlázquez, Isabel del Cursa-González, Ricardo Rodríguez-Barrientos, Aurora Barber-a-Martín et al. Effectiveness of an educational group intervention in primary healthcare for continued exclusive breastfeeding: PROLACT study. BMC Pregnancy and Childbrith. 2018; 18 (59): 1-10. Recuperado de: https://doi.org/10.1186/s12884-018-1679-3 